\title{
Inhibitory Effect on Lipid Accumulation: Comparison between Two Polymethoxylflavones, Tangeretin and Nobiletin, and One Flavonoid, Hesperetin, in 3T3-L1 Adipocytes
}

\author{
Jen-Yin Chen ${ }^{1,2}$, Chin Chen Chu ${ }^{2}$, Shih-Ying Chen ${ }^{3}$ and Pin Der Duh ${ }^{4 *}$ \\ ${ }^{1}$ Department of Senior Citizen Service Management, Chia Nan University of Pharmacy and Science, Taiwan \\ ${ }^{2}$ Department of Anesthesiology, Chi-Mei Medical Center, Taiwan \\ ${ }^{3}$ Department of Health and Nutrition, Chia Nan University of Pharmacy and Science, Taiwan \\ ${ }^{4}$ Department of Food Science and Technology, Chia Nan University of Pharmacy and Science, Taiwan
}

Received: February 27, 2018; Published: March 14, 2018

*Corresponding author: Pin Der Duh, Department of Food Science and Technology, Chia Nan University of Pharmacy and Science, 60 Erh-Jen Road, Section 1, Pao-An, Jen-Te District, Tainan, Taiwan, Tel: 886-6-2660993; Fax: 886-6-3663882; Email: ipdduh@mail.cnu.edu.tw

\begin{abstract}
In this study, two polymethoxylflavones (PMFs), including nobiletin and tangeretin, and one flavonoid, hesperetin with one methoxyl group, were investigated to inhibit lipid accumulation using 3T3-L1 adipocytes. The reducing lipid droplets of the three compounds at $50 \mu \mathrm{M}$ followed the order nobiletin=tangeretin>hesperetin; the inhibition of triglyceride levels was in the order nobiletin>tangeretin> hesperetin, indicating the numbers of methoxyl groups on the benzene ring play an important role in reducing lipid accumulation. These results imply polymethoxylflavones with high degree of methoxylation are easily permeable to cell membranes and demonstrate more regulating adipogenesis. The findings of this study are useful for designing new antiadipogenic agent and is therefore a potentially lipid lowering compounds based on chemical structure.
\end{abstract}

Keywords: Nobiletin; Tangeretin; Hesperetin; Polymethoxylflavone; Lipid Accumulation

Abbreviations: PMFs: Polymethoxylflavones; PPAR $\gamma$ : Peroxisome Proliferator-Activated Receptor $\gamma$; OROSM: Oil Red O Staining Material

\section{Introduction}

In 2016, more than 1.9 billion adults and 18 years and older were overweight, and over 340 million children and adolescents age 5-19 were overweight or obese [1]. Obesity is defined as abnormal or excess fat accumulation that may be closely associated with various human diseases, such as hypertension cardiovascular disease, type 2 diabetes mellitus, and certain cancers [2]. Adipocytes are the cells that primary compose adipose tissue. Thus, obesity results from the factor which adipocytes accumulates a large amount of fat and became enlarged [3]. Obesity and overweightness are the results of both hypertrophy, an increase in adipocyte size, and hyperplasia, an increase in adipocyte number [4]. Up to now there is growing body of evidence that a combination of behavioral, dietary and therapeutic approaches is required for effective prevention and treatment of obesity [4]. Polyphenolic compounds shown biological activities have been widely studied. Polymethoxylated flavones
(PMFs), having four or more methoxyl groups among flavonoids with a carbonyl group at the $\mathrm{C} 4$ position, have been of particular interest to their broad spectrum of biological activities, including antiallergic, anti-inflammatory and antioxidant activities [5]. Many studies have focused on two of the most abundant PMFs in citrus peels, particularly on nobiletin and tangeretin. For example, in mature 3T3-L1 adipocytes, nobiletin induced apoptosis; tangeretin suppressed triglyceride accumulation [6]. In addition, nobiletin exerts antiadipogenic effects through modulation of the PPAR and AMPK signaling pathway [7]. PMFs are more lipophic due to the hydrophobic character of methoxyl groups as compared to polyhydroxylated flavonoids [8]. Hesperetin is a flavonoid with trihydroxyflavone and an additional methoxyl substituent at the 4'-position. Many studies have revealed that PMFs are significantly more permeable to cell membranes than polyhydroxylated 
flavonoids; consequently, enhance their biological activities such as antiadipogenic activity. However, not much is known about the comparison between PMFs and polyhydroxylated flavonoid in antiadipogenic effect. The aim of this study was to compare the antiadipogenic effect of nobiletin and tangeretin, belonging PMFs, and hesperetin, a polyhydroxylated flavonoid with a methoxyl group, in 3T3-L1 cells.

\section{Materials and Methods \\ Chemicals}

Hesperetin was purchased from Sigma Chemical Co. (St. Louis, MO, USA). Tangeretin and nobiletin were obtained from Tokyo Chemical Industry (Toshima, Kita-ku, Tokyo, Japan). The chemical structures of the three compounds are shown in Figure 1, $[9,10]$ all chemicals were of analytical reagent grade.

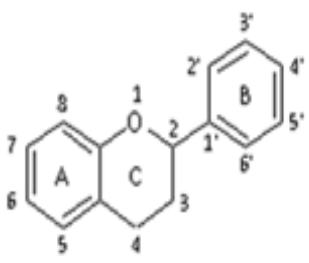

Flavonoid

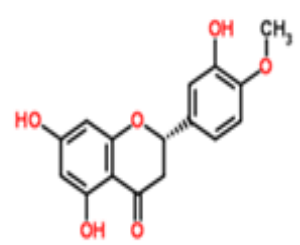

Hesperetin

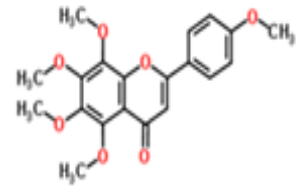

Tangeretin

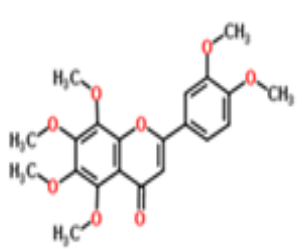

Nobiletin

Figure 1: Chemical structure of flavonoid, Hesperetin, Tangeretin and Nobiletin.

\section{Cell Cultural and Adipocytic Differentiation}

3T3-L1 preadipocytes (BCRC 60159) were purchased from Bioresources Collection and Research Center (Shin-Chu, Taiwan). Adipocytic differentiation was performed as described by the method of Hsu and Yen [3]. The tetrazolium dye colorimetric test (MTT test) was conducted to measure the cell viability [11].

\section{Measurement of Lipid Accumulation}

To monitor the lipid accumulation, Oil Red O Staining (OROSM) was conducted. 3T3-L1 cells were harvested 8 days after the initiation of differentiation. Experimental conditions were the same as those previously described [7]. All data are the average of triplicate analyses. In addition, intracellular triglyceride levels were also determined. The triglyceride levels were measured using a Triglyceride Colorimetric Assay Kit, (Cayman Chemical, Ann Arbor, MI, USA).

\section{Western Blot Analysis}

To understand peroxisome proliferator-activated receptor $\gamma$ (PPAR $\gamma$ ) expression of 3T3-L1 cells, Western blot analysis was conducted. In brief, on day 8, 3T3-L1 adipocytes were incubated with hesperetin, tangeretin and nobiletin for $24 \mathrm{~h}$ at $37^{\circ} \mathrm{C}$ in a humidified $5 \% \mathrm{CO}_{2}$ incubator. Primary antibodies against PPAR $\gamma$ and $\beta$-actin were applied at $4^{\mathrm{L}}$ movernight and then with secondary antibodies for $1 \mathrm{~h}$. The protein was detected using the WesternBright ECL kit (Advansta Inc., Menlo Park, CA, USA). The expression levels of PPAR $\gamma$ and $\beta$-actin proteins were determined by densitometry and analyzed [11].

\section{Results and Discussion}

At first, we examined the cell toxicity of the three compounds toward adipocytic 3T3-L1 cells using MTT assay. No significant difference on cell viability was found to the three compounds at
$50 \varangle \mathrm{M}$, indicating the three compounds in the range of tested concentrations show no toxicity toward adipocytic 3T3-L1 cells. Next, the cells were allowed to differentiate into adipocytes for 8 day and then the effects of the three compounds on lipid accumulation in 3T3-L1 adipocytes were measured. The effects of the three compounds on lipid accumulation in 3T3-L1 cells are shown in Figure 2. The Figure 2A shows the microscopic images of oil red 0 -stained adipocytes. The number of lipid droplets in the differentiated cells treated with the three compounds was decreased compared with the control. Quantitatively, OROSM showed hesperetin, tangeretin and nobiletin at $50 \otimes \mathrm{M}$ reduced lipid accumulation by 1.05, 35.52 and $35.86 \%$, respectively (Figure 2B), indicating the number of intracellular lipid droplets in the differentiated cells was marked reduced when tangeretin and nobiletin were present.

In addition, further analysis of the effects of the three compounds on the triglyceride levels are shown in Figure 2C. It was observed hesperetin, tangeretin and nobiletin at $50 \nabla \mathrm{M}$ reduced triglyceride levels in 3T3-L1 adipocytes by 16.08, 23.10 and $45.67 \%$, respectively, revealing the intracellular triglyceride levels in the differentiated cells treated with the three compounds were decreased compared with the control. Among the three compounds, nobiletin demonstrated the highest suppression of triglyceride levels. PPAR区 plays an important role in the induction and maintenance of the fully differentiated adipocyte phenotype [12]. Therefore, to understand whether three compounds inhibited lipid accumulation through regulating PPAR区, the PPAR囚 protein expression was conducted using Western blot analysis. As shown in Figure 2D, in the nobiletin-treated adipocytes, the PPAR区 expression was significantly inhibited, ilndicating nobiletin suppressed the cellular PPARष expression in the 3T3-L1 adipocytes. 

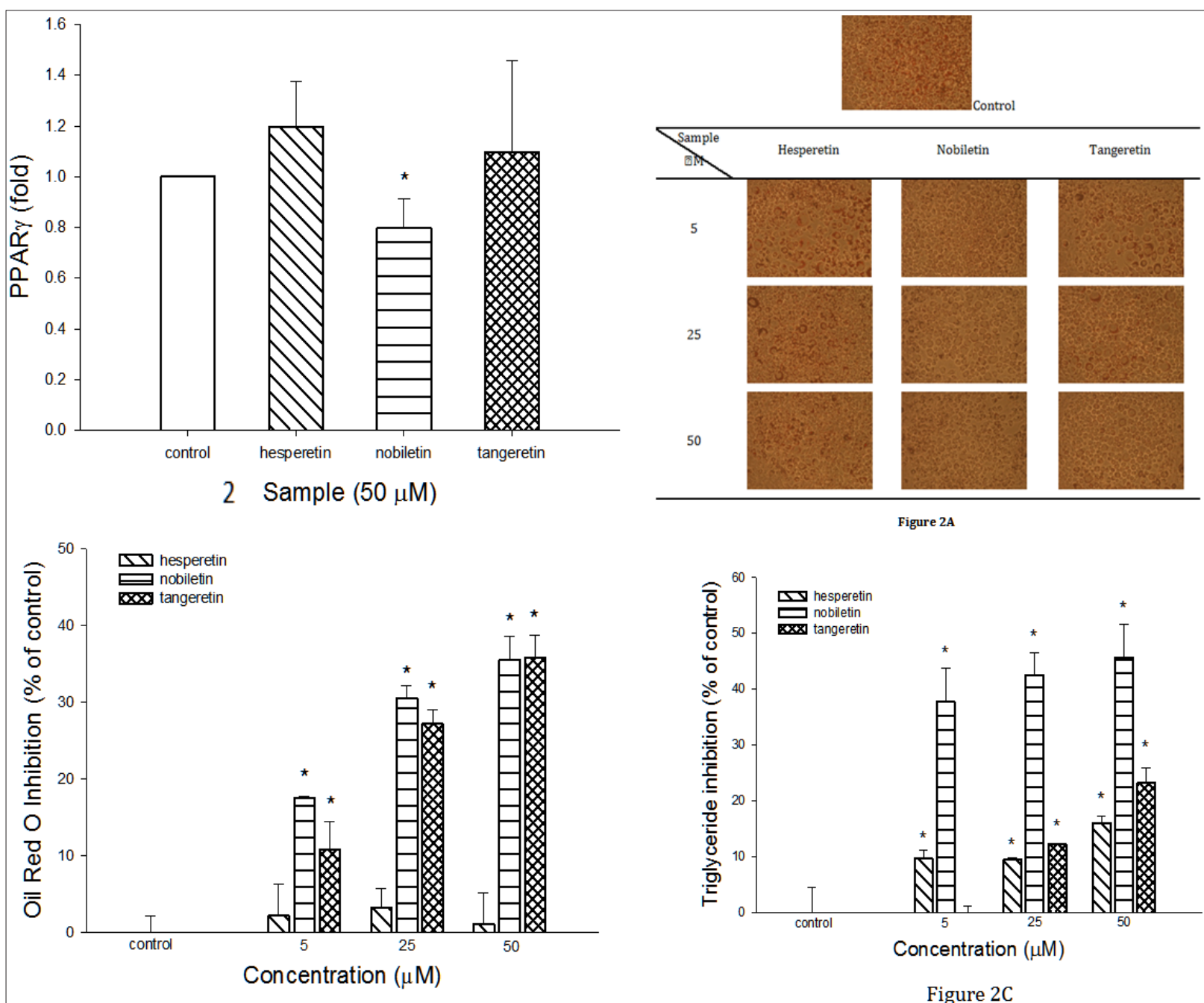

Figure $2 \mathrm{C}$

Figure 2B

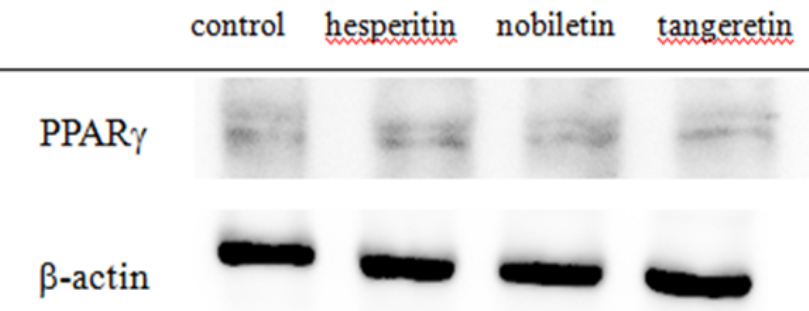

Figure 2D

Figure 2: The effects of hesperetin, tangeretin and nobiletin on lipid accumulation in 3T3-L1 adipocytes.

Figure 2A: The photographs of Oil red O-stainined adipocytes treated with or without hesperetin, tangeretin and nobiletin.

Figure 2B: Effects of hesperetin, tangeretin and nobiletin on Oil red O-stainined material (OROSM) in 3T3-L1 adipocytes.

Figure 2C: Hesperetin-, tangeretin- and nobiletin-mediated suppression of the intracellular triglyceride levels in 3T3-L1 adipocytes.

Figure 2D: The effect of hesperetin, tangeretin and nobiletin on protein levels of PPAR $\gamma$. Data are presented by means $\pm S D(n=3) .{ }^{*}(p<0.05)$ compared with the control group by Dunnett's test.

There was no significant difference in the cellular PPAR囚 expression between the control and the treatment with hesperetin and tangeretin. PPARX is an inducer of adipogenesis, which it can promote the transdifferentiation of cultured myoblasts to adipocytes, in particular, when coexpressed with C/EBPQ $[13,14]$.
In addition, PPAR区 plays an important role in the early stage of adipocyte differentiation, since it is a transcriptional factor for numerous genes [3]. Our current data shows nobiletin acted as a repressor of the expression of PPAR区. 
With respect to bioavailability of metabolisms of nobiletin, the methyl groups of the B-ring of nobiletin, particularly 4'-methyl group, are easily removed by methyltransferase enzyme [11,15]. Subsequently, the free hydroxyl groups can be conjugated by conjugation enzymes, and thereby resulting in increasing hydrophilicity and easily absorbed $[8,11]$. Consequently, demethylation of nobiletin facilitates its bioavailability, causing to increasing biological activity such as regulatory action toward adipocytes [11]. The biological activity of phenolic compounds is well associated with their structure [16]. The antimicrobial activity of PMFs are directed to their chemical structure, depending on the number and positions of hydroxyl groups and methoxyl groups, particularly, the presence of a lipophilic group at position 6 or 8 markedly improves biological activity [5].

Nobiletin, having the six methoxyl groups, located at the 3', 4',5,6,7,8 positions, and tangeretin with five methoxyl groups, located at the 4,5,6,7,8 positions, have high level of methoxylation and methoxyl groups at 6 and 8 positions, and thereby increasing the hydrophobic character of nobiletin and tangeretin. On the contrary, hesperetin, and flavonoid, has three hydroxyl groups at the position 3'-, 5-and 7-positions and a methoxyl substituent at the 4'-position. According the results, the reducing lipid droplets of the three compounds at $50 \nabla \mathrm{M}$ followed the descending sequence: nobiletin=tangeretin $>$ hesperetin; the inhibition of triglyceride levels was in the order nobiletin>tangeretin>hesperetin. In comparison with hesperetin, having a methoxyl group at the 4'-position, nobiletin and tangeretin showed higher reducing lipid accumulation, whereas hesperetin was relatively inactive. Apparently, the greater the number of methoxyl groups, the more inducing effect in regulation of adipogenesis. Therefore, we suggest nobilietin and tangeretin with high degree of methoxylation are easily permeable to cell membranes and can reach the active sites, therefore, showed more regulating adipogenesis.

\section{Conclusion}

In conclusion, the current data indicates PMFs such as nobiletin and tangeretin reduced lipid accumulation in vitro, and the regulatory activity against adipogenesis and the mode of action depended on the chemical structure. Consequently, multiple methoxyl groups of PMFs were suggested to the main role in regulating adipogenesis.

\section{Acknowledgement}

This research work was supported by Chi Mei Medical Center (CMFHR10613).

\section{References}

1. WHO (2017) World Health Organization, USA.

2. Fujimori K, Shibano M (2013) Avicularin, a plant flavonoid, suppresses lipid accumulation through repression of C/EBP $\alpha$-activated GLUT4mediated glucose uptake in 3T3-L1 cells. J Agric Food Chem 61(21): 5139-5147.

3. Hsu CL, Yen GC (2007) Effects of flavonoids and phenolic acids on the inhibition of adipogenesis in 3T3-L1 adipocytes. J Agric Food Chem 55(21): 8404-8410.

4. Sergeev IN, Li S, Ho CT, Rawson NE, Dushenkov S (2009) Polymethoxyflavones activate $\mathrm{Ca} 2+$-dependent apoptotic targets in adipocytes. J Agric Food Chem 57(13): 5771-5776.

5. Liu L, Xu X, Cheng D, Yao X, Pan S (2012) Structure-activity relationship of citrus polymethoxylated flavones and their inhibitory effects on Aspergillus niger. J Agric Food Chem 60(17): 4336-4341.

6. Miyata Y, Tanaka H, Shimada A, Sato T, Ito A, et al. (2011) Regulation of adipocytokine secretion and adipocyte hypertrophy by polymethoxyflavonoids, nobiletin and tangeretin. Life Sci 88(13-14): 613-618.

7. Choi Y, Kim Y, Ham H, Park Y, Jeong H S, et al. (2011) Nobiletin suppresses adipogenesis by regulating the expression of adipogenic transcription factors and the activation of AMP-activated protein kinase (AMPK). J Agric Food Chem 59(24): 12843-12849.

8. Li S, Lo CY, Ho CT (2006) Hydroxylated polymethoxyflavones and methylated flavonoids in sweet orange (Citrus sinensis) peel. J Agric Food Chem 54(12): 4176-4185.

9. (2014) Flavonoids: definition, structure and classification.

10. ChemSpider, USA.

11. Chu CC, Chen SY, Chyau CC, Fu ZH, Duh PD (2017) Antiproliferative effect of sweet orange peel and its bioactive compounds against human hepatoma cells, in vitro and in vivo. J Funct Foods 33: 363-375.

12. Gregoire FM, Smas CM, Sul HS (1998) Understanding adipocyte differentiation. Physiol Rev 78(3): 783-809.

13. Hu E, Tontonoz P, Spiegelman BM (1995) Transdifferentiation of myoblasts by the adipogenic transcription factors PPAR gamma and C/ EBP alpha. Proc Natl Acad Sci 92(21): 9856-9860.

14. Rosen ED, Walkey CJ, Puigserver P, Spiegelman BM (2000) Transcriptional regulation of adipogenesis. Genes Dev 14(11): 1293-1307.

15. Li S, Wang Z, Sang S, Huang MT, Ho CT (2006) Identification of nobiletin metabolites in mouse urine. Mol Nutr Food Res 50: 291-299.

16. Cai Y, Sun M, Xing J, Corke H (2004) Antioxidant phenolic constituents in roots of Rheum officinale and Rubia cordifolia: structure-radical scavenging activity relationships. J Agri Food Chem 52(26): 7884-7890. 
(c) (i) This work is licensed under Creative

Submission Link: https://biomedres.us/submit-manuscript.php

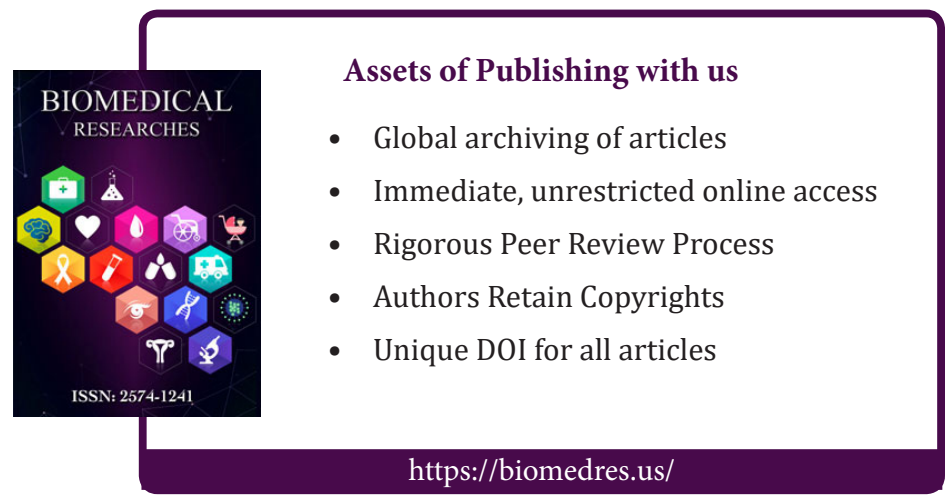

